\title{
Effect of prenatal and perinatal antibiotics on maternal health in Malawi, Tanzania, and Zambia
}

\section{Citation}

Aboud, Said, Gernard Msamanga, Jennifer S. Read, Lei Wang, Chelu Mfalila, Usha Sharma, Francis Martinson, Taha E. Taha, Robert L. Goldenberg, and Wafaie W. Fawzi. 2009. "Effect of Prenatal and Perinatal Antibiotics on Maternal Health in Malawi, Tanzania, and Zambia." International Journal of Gynecology \& Obstetrics 107 (3) (December): 202-207. doi:10.1016/ j.ijgo.2009.07.037.

\section{Published Version}

doi:10.1016/j.ijgo.2009.07.037

\section{Permanent link}

http://nrs.harvard.edu/urn-3:HUL.InstRepos:27001570

\section{Terms of Use}

This article was downloaded from Harvard University's DASH repository, and is made available under the terms and conditions applicable to Other Posted Material, as set forth at http:// nrs.harvard.edu/urn-3:HUL.InstRepos:dash.current.terms-of-use\#LAA

\section{Share Your Story}

The Harvard community has made this article openly available.

Please share how this access benefits you. Submit a story.

Accessibility 


\title{
Effect of prenatal and perinatal antibiotics on maternal health in Malawi, Tanzania, and Zambia
}

\author{
Said Aboud ${ }^{a},{ }^{*}$, Gernard Msamanga ${ }^{b}$, Jennifer S. Read ${ }^{c}$, Lei Wang ${ }^{d}$, Chelu Mfalilad, Usha \\ Sharma $^{e}$, Francis Martinson ${ }^{f}$, Taha E. Tahag, Robert L. Goldenberg ${ }^{h}$, and Wafaie W. Fawzi ${ }^{i}$ \\ aDepartment of Microbiology and Immunology, Muhimbili University of Health and Allied Sciences, \\ Dar es Salaam, Tanzania \\ ${ }^{b}$ Department of Community Health, Muhimbili University of Health and Allied Sciences, Dar es \\ Salaam, Tanzania \\ 'Pediatric, Adolescent, and Maternal AIDS Branch, NICHD, NIH, DHHS, Bethesda, MD, USA \\ dStatistical Center for HIV/AIDS Research and Prevention (SCHARP), Fred Hutchinson Cancer \\ Research Center (FHCRC), Seattle, WA, USA \\ ePrevention Sciences Program, NIAID, NIH, Bethesda, MD, USA \\ fUNC Project, Lilongwe, Malawi \\ gDepartment of Epidemiology, Johns Hopkins University Bloomberg School of Public Health, \\ Baltimore, MD, USA \\ hDepartment of Obstetrics and Gynecology, Drexel University College of Medicine, Philadelphia, \\ PA, USA \\ 'Department of Nutrition, Harvard School of Public Health, Boston, MA, USA
}

\section{Abstract}

Objective-We assessed the effect of prenatal and peripartum antibiotics on maternal morbidity and mortality among HIV-infected and uninfected women.

\begin{abstract}
Methods-A multicenter trial was conducted at clinical sites in 4 Sub-Saharan African cities: Blantyre and Lilongwe, Malawi; Dar es Salaam, Tanzania; and Lusaka, Zambia. A total of 1558 HIV-infected and 271 uninfected pregnant women who were eligible to receive both the prenatal and peripartum antibiotic/placebo regimens were enrolled. Pregnant women were interviewed at 20-24 weeks of gestation and a physical examination was performed. Women were randomized to receive either antibiotics or placebo. At the 26-30 week visit, participants were given antibiotics or placebo to be taken every 4 hours beginning at the onset of labor and continuing after delivery 3 times a day
\end{abstract}

\footnotetext{
(C) 2009 International Federation of Gynecology and Obstetrics. Published by Elsevier Ireland Ltd. All rights reserved.

*Corresponding author: S. Aboud, Department of Microbiology and Immunology, Muhimbili University of Health and Allied Sciences, P.O. Box 65001, Dar es Salaam, Tanzania. Tel: +255 222150302 ext. 276; Fax: +255 22 2153114. aboudsaid@ yahoo.com.

Publisher's Disclaimer: This is a PDF file of an unedited manuscript that has been accepted for publication. As a service to our customers we are providing this early version of the manuscript. The manuscript will undergo copyediting, typesetting, and review of the resulting proof before it is published in its final citable form. Please note that during the production process errors may be discovered which could affect the content, and all legal disclaimers that apply to the journal pertain.

Conflict of interest

The conclusions and opinions expressed in this paper are those of the authors and do not necessarily reflect those of the funding agencies and participating institutions. The authors declare that there are no conflicts of interests.
} 
until a 1-week course was completed. Logistic regression and Cox proportional hazards models were used.

Results-There were no significant differences between the antibiotic and placebo groups for medical conditions, obstetric complications, physical examination findings, puerperal sepsis, and death in either the HIV-infected or the uninfected cohort.

Conclusion-Administration of study antibiotics during pregnancy had no effect on maternal morbidity and mortality among HIV-infected and uninfected pregnant women.

\section{Keywords}

Antibiotics; HIV; Maternal morbidity; Maternal mortality; Pregnancy

\section{Introduction}

Pathogenic bacteria that are common inhabitants of the vagina may cause infections such as cervicitis, chorioamnionitis, and intra-amniotic infection during pregnancy. For example, bacterial vaginosis is a common condition in which the normal vaginal lactobacilli are replaced by a mixture of bacteria including Gardnerella vaginalis, Mobiluncus spp, Bacteroides spp, and Mycoplasma hominis. These same organisms are frequently found in intrauterine infections, especially of the chorioamnion. Such infections are known to have an important role either as a cause or a consequence of preterm labor or premature rupture of membranes (PROM) and subsequent preterm birth [1,2].

Antibiotic prophylaxis given during the second and third trimesters of pregnancy may prevent or treat such infections and hence reduce the risk of preterm labor or PROM when given routinely to pregnant women. In a randomized clinical trial of antibiotics in women at risk of endometritis in United States, administration of cefotetan and doxycycline/erythromycin resulted in a reduced frequency of postpartum endometritis [3]. Similarly, in a recent randomized controlled trial of prophylactic antibiotics to prevent postpartum infectious morbidity in HIV-infected women who delivered vaginally, a single intrapartum prophylactic dose of cefoxitin was shown to significantly reduce (13.6\% in placebo vs $6.4 \%$ in antibiotic group) postpartum endometritis [4]. Other antibiotic studies showed no benefit in reducing maternal morbidity [5-7]. A trial of antibiotics using metronidazole, erythromycin, and ampicillin (HPTN 024 study) conducted among HIV-infected and uninfected pregnant women to prevent chorioamnionitis-associated mother-to-child transmission (MTCT) of HIV and preterm birth showed that these antibiotics did not reduce either the risk of MTCT of HIV or the rate of preterm birth $[8,9]$. However, there is a relative paucity of research on whether these antibiotic regimens affect maternal health outcomes, particularly in populations with HIV-1 infection. The aim of the present study was to investigate the effects of antibiotic administration prenatally and during labor on maternal morbidity and mortality among HIV-infected and uninfected pregnant women enrolled in the HPTN 024 study.

\section{Materials and methods}

HPTN 024 was a randomized, double-blinded, placebo-controlled Phase III trial that enrolled both HIV-infected and HIV-uninfected women. The primary objectives of this trial were to determine the efficacy of a low-cost antibiotic intervention for prevention of chorioamnionitisassociated MTCT of HIV and of preterm birth [8]. This multicenter trial was conducted at clinical sites in 4 Sub-Saharan African cities: Blantyre and Lilongwe, Malawi; Dar es Salaam, Tanzania; and Lusaka, Zambia. Enrollment started in July 2001 and ended in February 2003. The design and results of the HPTN 024 trial have been described in detail elsewhere [8,9]. Briefly, pregnant women (between 20 and 24 weeks of pregnancy) were randomized to receive 
either antibiotics ( $250 \mathrm{mg}$ of metronidazole 3 times a day and $250 \mathrm{mg}$ of erythromycin orally 3 times a day for 7 days) or placebo. The antibiotics and dosages were chosen because they were generally effective against the vaginal and intrauterine organisms and reduction in preterm birth may be achieved using a prolonged course of antibiotics (1 week or more) in the second trimester. Data regarding socio-demographic characteristics as well as obstetric and medical history were obtained through participant interviews. The external genitalia, vagina, and cervix were examined, and a bimanual examination was performed. In addition to physical examination, laboratory tests were performed (including HIV testing, quantification of CD4 cell counts and plasma viral loads, and screening for syphilis, trichomoniasis, candidiasis, bacterial vaginosis, gonorrhea and Chlamydia infection) [8]. In addition, at the 26-30 week visit, participants were given antibiotics (250 $\mathrm{mg}$ of metronidazole and $500 \mathrm{mg}$ of ampicillin) or placebo to be taken at the onset of labor and then every 4 hours, continuing after delivery 3 times a day until the 1-week course was completed. Compliance with treatment was assessed using pill counts at the 26-30 week visit as described previously [8]. Women were asked to bring the peripartum antibiotics/placebo blister packs to the next study visit at 4-6 weeks post partum. Adherence was assessed by the number of pills missing from the blister pack at the follow-up visit. If the blister packs were not returned, reported use was noted. Adherence to prenatal study drugs was assessed among 1562 (99\%) of the 1573 women randomly assigned and delivered before March 5, 2003. Of these, 98\% in each study arm took all 21 tablets of metronidazole and erythromycin prenatally. Adherence was also high at the time of labor: 1392 (88\%) of the 1573 women took at least one dose before delivery. Physical examinations during pregnancy, at delivery, discharge, and within 3 months after delivery were performed to determine the mothers' medical conditions and obstetric complications. The HPTN 024 trial revealed that, despite reducing vaginal infections, the antibiotic regimen used did not reduce preterm birth, increase the time to delivery, or increase birth weight [9]. HIV-infected women and their infants were offered MTCT prophylaxis with nevirapine [10]. Mothers' medical conditions and obstetric complications (including postpartum hemorrhage) were managed according to the existing obstetrics management guidelines in the individual countries (including the use of active management of the third stage of labor and use of oxytocic agents). Data presented at an interim Data Safety Monitoring Board (DSMB) showed that the antibiotic regimen did not show a meaningful reduction of MTCT of HIV. Thus, further randomization and distribution of study drugs was halted on March 5, 2003 at all study sites.

Because of the early termination of the trial by the DSMB, this analysis includes only those HIV-infected and uninfected pregnant women who were eligible to receive both the prenatal and peripartum antibiotic/placebo regimens. These were the women who were randomized on or before October 15, 2002. This date was determined by subtracting 140 days from March 5, 2003 , assuming a woman entered the study at a gestational age of 20 weeks and the normal course of pregnancy is 40 weeks.

We examined the effect of antibiotics versus placebo on a number of maternal medical conditions and physical examination findings including diarrhea (3 or more loose and watery stools per day over the last 2 weeks); cough (coughing all day for more than 2 weeks); fever (hot to touch for more than 2 weeks); recurrent itchy vaginal discharge; herpes zoster; oral thrush; hospitalization; weight gain or loss during pregnancy; mastitis; puerperal sepsis; and death. Information on diarrhea, cough, fever, itchy vaginal discharge, and hospitalization was collected at enrollment, the first and second prenatal visits, the intrapartum visit, and followup visits post delivery. Data regarding fever during labor or post partum; prenatal or postpartum hemorrhage; and hypertension with or without proteinuria were collected at the intrapartum visit. Information on puerperal sepsis and admission to the postnatal ward was collected at the time of maternal discharge from the hospital. Weight was measured at enrollment, the first and second prenatal visits, and at discharge. Mastitis was defined as inflammation of the breast 
occurring within 3 months post delivery. Data related to syphilis risk factors, prevalence, and birth outcomes in the HPTN 024 cohort have been published [11].

This was a planned secondary analysis. The sample size in the original study design (i.e. 1560 women in each arm) would provide adequate power to detect a medium or large effect in the target medical conditions between the treatment arms. With the sample size used in this analysis (i.e. $779 \mathrm{HIV}$-infected women in each arm), the power is less adequate, especially when the medical condition is rare or the expected effect size is small. To assess differences in baseline characteristics (including demographics, laboratory results, obstetric and medical history, and medical conditions/physical examination findings) between the antibiotic and the placebo arms, Pearson $\chi^{2}$ or Fisher exact test $P$ values were reported for categorical variables, and 2sample $t$ test or Wilcoxon rank sum test $P$ values were calculated for continuous variables. For medical conditions for which data were collected on multiple occasions, generalized estimating equations (GEE) models were used to compute the odds of having a condition in the antibiotics arm versus the placebo arm, while adjusting for baseline conditions. A logistic regression model was utilized to analyze binary outcomes that were collected only once during followup. A Cox proportional hazards model was used to estimate treatment effect on death. Weight gain was defined as the average per-week weight change from enrollment to the second prenatal visit or the first one thereafter if a woman missed her second visit. A general linear model was used to ascertain the effect of treatment on weight gain adjusting for gestational age at enrollment (measured by fundal height in centimeters). Analyses on the effect of the intervention were performed separately for HIV-infected and uninfected cohorts. $P<0.05$ was regarded as statistically significant. All statistical analyses were performed using SAS version 9.1.3 on SunOS 5.9 platform (SAS, Cary NC, USA).

Written informed consent was obtained from all participants prior to HIV testing and inclusion in the study. The study protocol was approved by U.S. institutional review boards (Bloomberg School of Public Health, Johns Hopkins University, Baltimore, MD; School of Medicine, University of Alabama at Birmingham; School of Medicine, University of North Carolina at Chapel Hill; Harvard University School of Public Health, Boston, MA) and by institutional ethical review boards/committees (University of Malawi College of Medicine, Lilongwe, Malawi; Muhimbili University College of Health Sciences, Dar es Salaam, Tanzania; and the University of Zambia School of Medicine, Lusaka, Zambia).

\section{Results}

Of the 2659 women enrolled in HPTN 024, 1829 women (1558 HIV-infected and 271 HIVuninfected) met the inclusion criteria for this analysis (Figure 1). Baseline characteristics of women by randomization arms were comparable in both the HIV-infected cohort (Table 1) and the HIV-uninfected cohort (Table 2).

There were no statistically significant differences observed in the occurrence of clinical conditions such as mastitis, diarrhea in the previous 2 weeks, fever or cough for more than 2 weeks in the last 3 months, vaginal discharge or herpes zoster in the last 3 months, oral thrush in the last month, or hospitalization between the placebo and the antibiotic arms in either the HIV-infected or the HIV-uninfected cohorts within 3 months after delivery (Table 3).Eighteen deaths occurred in the HIV-infected cohort, 10 in the antibiotics arm and 8 in the placebo arm. A non-significant increase in the survival time was observed in the antibiotic group, either stratifying (Hazard ratio 1.23; 95\% CI, 0.49-3.12) or not stratifying by site (Hazard ratio 1.22; 95\% CI, 0.49-3.08). No deaths occurred in the HIV-uninfected cohort. There was no significant difference in average weight gain during pregnancy between the antibiotic and placebo arms in either the HIV-infected ( $0.29 \mathrm{vs} 0.26 \mathrm{~kg} ; P=0.12)$ or the HIV-uninfected $(0.29 \mathrm{vs} 0.31 \mathrm{~kg}$; $P=0.77$ ) cohort. 


\section{Discussion}

Pregnancy is a physiological state in which immune function is expected to be compromised, particularly in HIV-infected women. Therefore, preventing or treating subclinical infections could have a beneficial effect on maternal and infant morbidity and mortality. However, the results of the current analysis indicate there are no significant differences in morbidity or mortality in either the HIV-infected or HIV-uninfected cohorts according to randomization arm (antibiotics vs placebo). Similarly, there was no significant difference in average weight gain during pregnancy between the antibiotic and placebo arms.

Several trials have been conducted previously with various chemoprophylactic regimens to determine their effects on pregnancy outcomes and neonatal morbidity. A relatively successful application of chemoprophylaxis has been seen in prolongation of the latency period between the rupture of membranes and delivery, thus increasing the gestational age at birth $[3,12,13]$. Results of the HPTN 024 trial showed that there were few differences in mean gestational age at delivery, the percentage of preterm births, the time between randomization and delivery, or birth weight between antibiotic- versus placebo-treated HIV-infected and uninfected women [9]. Four weeks after the 24-week antibiotic/placebo course, bacterial vaginosis and trichomoniasis were reduced by $49 \%$ to $61 \%$ in the antibiotic compared with the placebo groups [9]. Overall the antibiotics used did not show benefit in reducing MTCT or preterm birth.

Our study findings showed that there was no effect of antibiotics on mastitis and pyrexia in HIV-infected women. Similar findings have been reported previously [4]. In the current study, metronidazole and erythromycin were given at 20 to 24 weeks of gestation for 7 days while metronidazole and ampicillin were taken during labor through 1 week post partum. In the previous study a single dose of cefoxitin was given intrapartum at 36 weeks or more of gestation [4]. Possible effects of other co-interventions including the use of active management of the third stage of labor and oxytocic agents could possibly have impacts on the rates of postpartum hemorrhage and also indirectly on puerperal sepsis. These interventions may reduce the statistical power of our analyses, but since the patients were randomized this would not affect the validity of results of the trial. In addition, we found no effect of antibiotics on other maternal morbidity or mortality. Our study findings may suggest that since pregnancy is associated with weight gain, and increases of intravascular volume, glomerular filtration, and hepatic metabolism, $250 \mathrm{mg}$ of erythromycin orally 3 times a day administered for 7 days may have not been sufficient for infection prophylaxis during pregnancy. In addition, maternal plasma levels of antibiotics reach values $10 \%-15 \%$ under the ranges observed with the same dosages administered to non-pregnant women [14].

A limitation of our analyses on the efficacy of antibiotics on maternal health outcomes is the relatively small number of events for the medical and obstetric endpoints. This is likely to compromise the statistical power to detect real differences between the antibiotic and the placebo groups. Future research with a larger sample sizes as well as different and potentially more effective yet safe antibiotic regimens is, therefore, needed to further elucidate the effect of antibiotic use on maternal health outcomes.

It is concluded that administration of study antibiotics during pregnancy had no effect on amaternal morbidity and mortality among HIV-infected and uninfected pregnant women.

\section{Acknowledgments}

The HPTN 024 trial was supported by the HIV Prevention Trials Network (HPTN) and sponsored by the U.S. National Institute of Allergy and Infectious Diseases (NIAID), National Institutes of Health, Department of Health and Human Services, through contracts N01-AI-035173, N01-AI-045200, and N01-AI-035173-117/412. In addition, the trial was supported by the HIV Prevention Trials Network (HPTN) and sponsored by NIAID; Eunice Kennedy Shriver National 
Institute of Child Health and Human Development; National Institute on Drug Abuse; the National Institute of Mental Health; and the Office of AIDS Research, of the National Institutes of Health, U.S. Department of Health and Human Services, through Harvard University (U01-AI-048006); Johns Hopkins University (U01-AI-048005); and the University of Alabama at Birmingham (U01-AI-047972). Support for this work was also provided by the AIDS International Training and Research Program (D43 TW01035-07).

\section{References}

1. Parry S, Strauss JF 3rd. Premature rupture of the fetal membranes. N Engl J Med 1998;338(10):663670. [PubMed: 9486996]

2. Goldenberg RL, Andrews WW, Yuan A, MacKay T, St Louis M. Sexually transmitted diseases and adverse outcomes of pregnancy. Clinics in Perinatology 1997;24:23-41. [PubMed: 9099500]

3. Andrews WW, Hauth JC, Cliver SP, Savage K, Goldenberg RL. Randomized clinical trial of extended antibiotic spectrum with coverage for Ureaplasma urealyticum to reduce post-cesarean delivery endometritis. Obstet Gynecol 2003;101(6):1183-1189. [PubMed: 12798523]

4. Sebitloane HM, Moodley J, Esterhuizen TM. Prophylactic antibiotics for the prevention of postpartum infectious morbidity in women infected with human immunodeficiency virus: a randomized controlled trial. Am J Obstet Gynecol 2008;198(2):e1-e6. [PubMed: 17997391]189

5. Andrews WW, Sibai BM, Thom EA, Dudley D, Ernest JM, McNellis D, et al. Randomized clinical trial of metronidazole plus erythromycin to prevent spontaneous preterm delivery in fetal fibronectinpositive women. Obstet Gynecol 2003;101(5 Pt 1):847-855. [PubMed: 12738139]

6. Thigpen BD, Hood WA, Chauhan S, Bufkin L, Bofill J, Magann E, et al. Timing of prophylactic administration in the uninfected laboring gravida: a randomized clinical trial. Am J Obstet Gynecol 2005;192(6):1864-1871. [PubMed: 15970833]

7. Ovalle A, Romero R, Gomez R, Martinez MA, Nien JK, Ferrand P, et al. Antibiotic administration to patients with preterm labor and intact membranes: is there a beneficial effect in patients with endocervical inflammation. J Matern Fetal Neonatal Med 2006;19(8):453-464. [PubMed: 16966109]

8. Taha TE, Brown ER, Hoffman IF, Fawzi W, Read JS, Sinkala M, et al. A phase III clinical trial of antibiotics to reduce chorioamnionitis-related perinatal HIV-1 transmission. AIDS 2006;20(9):13131321. [PubMed: 16816561]

9. Goldenberg RL, Mwatha A, Read JS, Adeniyi-Jones S, Sinkala M, Msamanga G, et al. The HPTN 024 Study: the efficacy of antibiotics to prevent chorioamnionitis and preterm birth. Am J Obstet Gynecol 2006;194(3):650-661. [PubMed: 16522393]

10. Guay LA, Musoke P, Fleming T, Bagenda D, Allen M, Nakabiito C, et al. Intrapartum and neonatal single-dose nevirapine compared with zidovudine for prevention of mother-to-child transmission of HIV-1 in Kampala, Uganda: HIVNET 012 randomized trial. Lancet 1999;354(9181):795-802. [PubMed: 10485720]

11. Potter D, Goldenberg RL, Read JS, Wang J, Hoffman IF, Saathoff E, et al. Correlates of syphilis seroreactivity among pregnant women: the HIVNET 024 Trial in Malawi, Tanzania, and Zambia. Sex Transm Dis 2006;33(10):604-609. [PubMed: 16601659]

12. Mercer BM, Miodovnik M, Thurnau GR, Goldenberg RL, Das AF, Ramsey RD, et al. Antibiotic therapy for reduction of infant morbidity after preterm premature rupture of the membranes. A randomized controlled trial. National Institute of Child Health and Human Development MaternalFetal Medicine Units Network. JAMA 1997;278(12):989-995. [PubMed: 9307346]

13. Thinkhamrop J, Hofmeyr GJ, Adetoro O, Lumbiganon P. Prophylactic antibiotic administration in pregnancy to prevent infectious morbidity and mortality. Cochrane Database Syst Rev 2002:CD002250. [PubMed: 12519571]

14. Bossens M. Antibiotics and pregnancy. Rev Med Brux 2001;22(4):A260-A263. [PubMed: 11680185] 


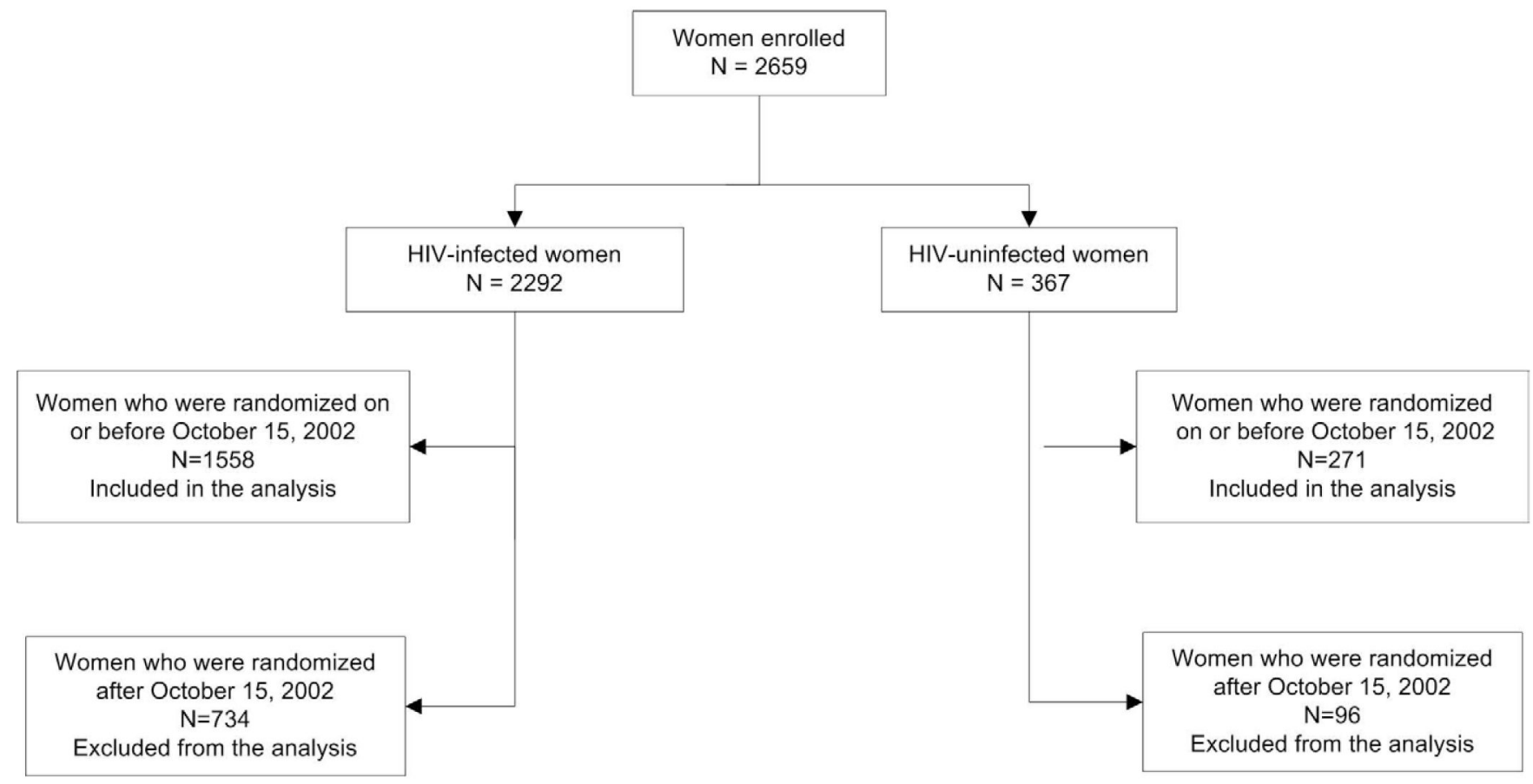

Figure 1.

Derivation of the study population 


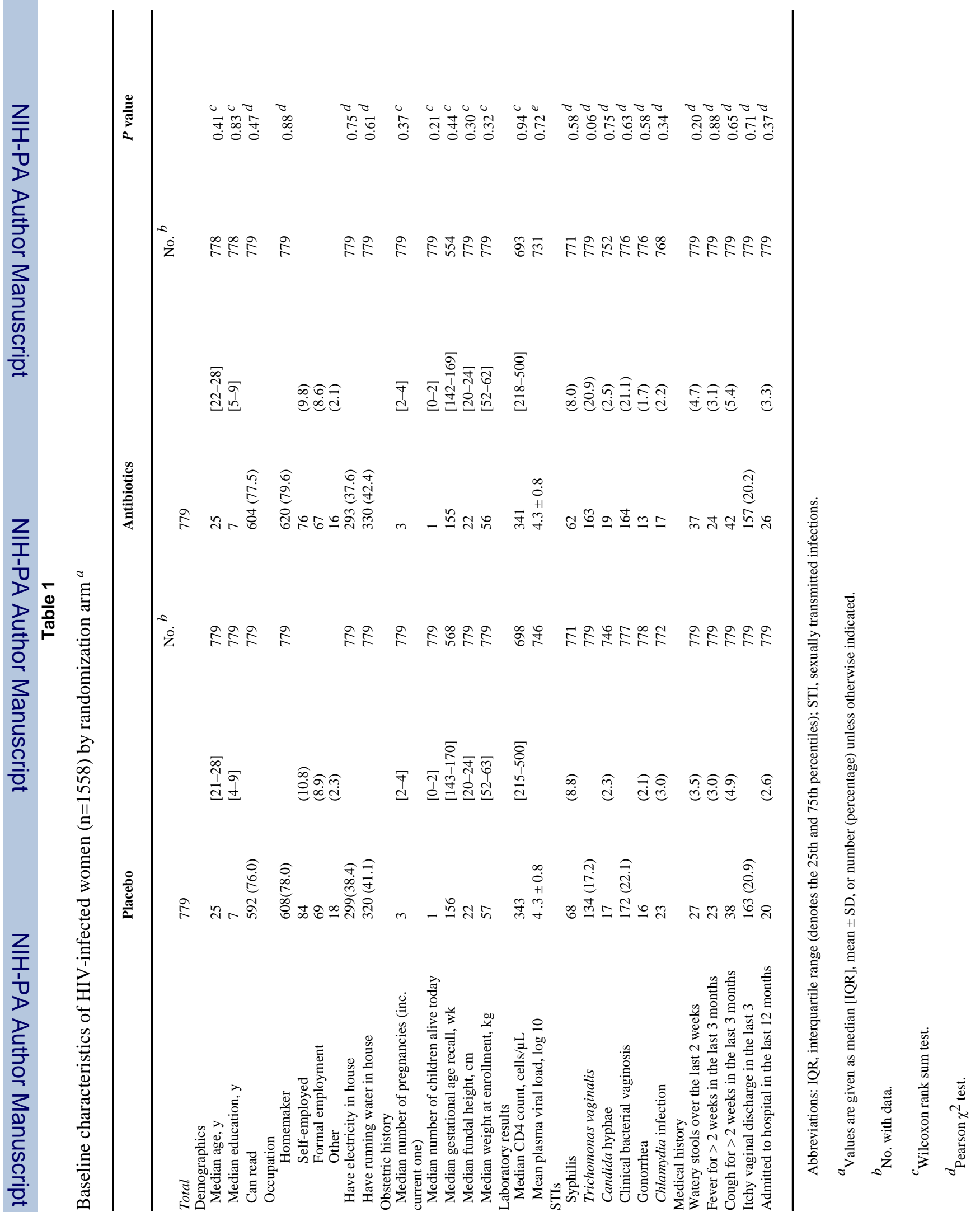




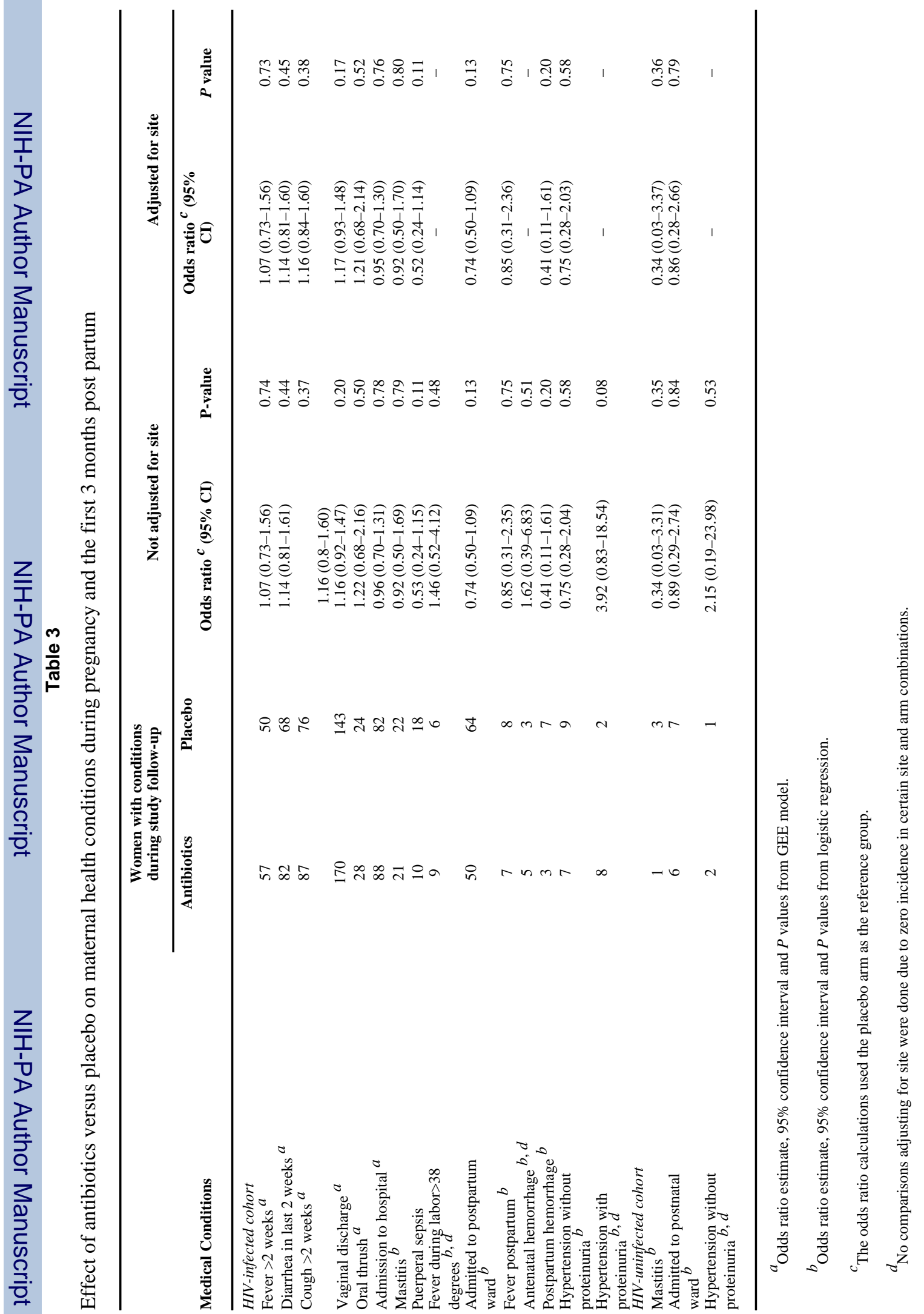

Int J Gynaecol Obstet. Author manuscript; available in PMC 2010 December 1. 